\title{
Transcriptional Expression Changes During Compensatory Plasticity in the Central Nervous System of the Adult Cricket Gryllus Bimaculatus: I. Auditory System Plasticity in the Prothoracic Ganglia
}

Felicia Wang

Bowdoin College

Harrison Fisher

Bowdoin College

Maeve Morse

Bowdoin College

Lisa L. Ledwidge

Bowdoin College

Jack O'Brien

Bowdoin College

Sarah E . Kingston

University of Maine

Justin Beckman

Bowdoin College

Jasmine J. Johnson

Bowdoin College

Lyn S. Miranda Portillo

Bowdoin College

Tabarak N Al Musawi

Bowdoin College

Hadley Wilson Horch ( $\nabla$ hhorch@bowdoin.edu )

Bowdoin College

Research Article

Keywords: RNASeq, Transcriptome, Dendritic Plasticity, GO term analysis, Differential expression, Injury

Posted Date: February 19th, 2021 
DOI: https://doi.org/10.21203/rs.3.rs-211260/v1

License: (c) (1) This work is licensed under a Creative Commons Attribution 4.0 International License. Read Full License 


\section{Transcriptional expression changes during compensatory}

2

3

22 Keywords:

23 RNASeq, Transcriptome, Dendritic Plasticity, GO term analysis, Differential expression, Injury

plasticity in the central nervous system of the adult cricket

\author{
Gryllus bimaculatus
}

\section{Auditory system plasticity in the prothoracic ganglia}

Felicia Wang, Harrison Fisher, Maeve Morse, Lisa L. Ledwidge, Jack O’Brien, Sarah E.

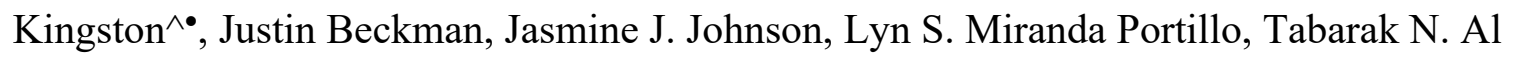

Musawi, Hadley Wilson Horch*,

Department of Biology, Bowdoin College, 6500 College Station, Brunswick, Maine 04672 USA.

${ }^{\wedge}$ School of Marine Sciences and Darling Marine Center, University of Maine, 193 Clarks Cove Rd., Walpole, ME 04573 USA;

-University of California Santa Cruz, Ecology and Evolutionary Biology Department and UC Natural Reserves, 1156 High St, Santa Cruz, CA 95064 USA

*Correspondence to:

Dr. Hadley Wilson Horch, Department of Biology, Bowdoin College 6500 College Station, Brunswick ME 04011 USA. Phone: 207-798-4128; FAX: 207-725-3405; Email:

hhorch@bowdoin.edu 


\section{Abstract}

28 Most adult organisms are limited in their capacity to recover from neurological damage. The

29 auditory system of the Mediterranean field cricket, Gryllus bimaculatus, presents a compelling

30 model for investigating neuroplasticity due to its unusual capabilities for structural

31 reorganization into adulthood. Specifically, the dendrites of the central auditory neurons of the

32 prothoracic ganglion sprout in response to the loss of auditory afferents. Deafferented auditory

33 dendrites grow across the midline, a boundary they normally respect, and form functional

34 synapses with the contralateral auditory afferents, restoring tuning-curve specificity. The

35 molecular pathways underlying these changes are entirely unknown. Here, we used a multiple k-

36 mer approach to re-assemble a previously reported prothoracic ganglion transcriptome that

37 included ganglia collected one, three, and seven days after unilateral deafferentation in adult,

38 male animals. We used EdgeR and DESeq2 to perform differential expression analysis and we

39 examined Gene Ontologies to further understand the potential molecular basis of this

40 compensatory anatomical plasticity. Enriched GO terms included those related to protein

41 translation and degradation, enzymatic activity, and Toll signaling. Extracellular space GO terms

42 were also enriched and included the upregulation of several protein yellow family members one

43 day after deafferentation. Investigation of these regulated GO terms help to provide a broader

44 understanding of the types of pathways that might be involved in this compensatory growth and

45 can be used to design hypotheses around identified molecular mechanisms that may be involved

46 in this unique example of adult structural plasticity. 


\section{Background}

Most adult organisms, especially mammals, are limited in their capacity to adapt and

51 recover from neurological damage $(1,2)$. The Mediterranean field cricket, Gryllus bimaculatus,

52 provides a model of neuroplasticity due to its demonstrated ability to compensate for neuronal

53 damage with novel dendritic growth and synapse formation, even into adulthood. Specifically,

54 the central auditory system, much of which resides in the prothoracic ganglion, reorganizes in

55 response to deafferentation caused by unilateral transection of auditory afferents in the adult (3$565)$.

In G. bimaculatus, auditory information is transduced by the auditory organs, located on

the prothoracic limbs. Auditory afferents receive the sensory stimuli and convey this information

59 into the prothoracic ganglion where they form synapses with several different auditory neurons

$60(6,7)$. These neurons exist as mirror image pairs and their dendritic arbors remain localized

61 ipsilateral to the auditory input, typically not projecting contralaterally across the midline (8).

62 However, previous research has shown that after amputation of the prothoracic leg in the adult,

63 which removes the auditory organ and severs the afferents, the deafferented dendrites of the

64 ipsilateral auditory neurons sprout across the midline and form functional synapses with the

65 intact auditory afferents on the contralateral side. This reorganization is evident whether

66 deafferentation occurs in larvae $(9,10)$ or adults $(3-5)$. Various aspects of the physiological

67 consequences of this compensatory behavior have been studied $(3,9,10)$, however little is known

68 about the molecular pathways and mechanisms underlying this growth.

69 Although the genome has only just become publicly available (11), various de novo

70 transcriptomes have been created for use in this species (12-14). Recently, a de novo

71 transcriptome of the prothoracic ganglion was assembled in an attempt to understand the 
72 molecular basis of the compensatory response (15). This transcriptome was built with RNA from

73 individual prothoracic ganglia of both control and deafferented adult male crickets. Initially, this

74 transcriptome was assembled and mined for the presence of developmental guidance molecules.

75 These guidance molecules are known to play a well-conserved role in regulating the specific

76 growth of axonal and dendritic projections during the development of many species $(16,17)$.

77 While these molecules have mainly been studied for their role in development, it has also been

78 suggested that alterations in their expression may influence the ability of axons and dendrites to

79 recover from injury in adulthood (15,18-20). Mining this cricket transcriptome revealed that

80 many well-conserved developmental guidance molecules, including slit, ephrins, netrins, and

81 semaphorins, were present within the adult prothoracic transcriptome (15). However, it is still

82 unknown whether the expression of these transcripts, or any other transcripts, are significantly

83 altered during this compensatory growth process.

84 The goal of this study was to better understand the underlying molecular control of the

85 compensatory growth behavior observed in the cricket. We assembled a new, more

86 representative and less redundant transcriptome of the cricket prothoracic ganglion using

87 multiple k-mer values during the assembly process. We also utilized the Evidential Gene

88 tr2aacdsmRNA classifier to reduce redundancies (21). This new transcriptome was used to

89 analyze changes in expression levels one, three, and seven days post-deafferentation. The

90 identified genes were then analyzed using GO annotation analysis to determine the classes of

91 genes that are differentially regulated over the course of the injury response. By performing this

92 analysis, it was possible to discover changes in gene expression that occur during the

93 compensatory growth response, allowing for insights into possible pathways or key molecules

94 critical to this process. 


\section{Results and Discussion}

\section{Transcriptome Assembly and Analysis}

97 This transcriptomic study focused on the cricket, Gryllus bimaculatus, whose nervous system has

98 been shown to have an unusual level of adult structural plasticity (3-5). We deafferented

99 sensory neurons, including the auditory neurons, in the prothoracic ganglion of the adult cricket,

100 by unilateral amputation of the prothoracic leg at the femur. Control amputations consisted of

101 removal of the distal tip of the tarsus. We harvested prothoracic ganglia one, three, and seven

102 days post-amputation. These time points were designed to capture transcriptional changes in

103 response to the injury (one day), during initial sprouting (one and three days), growth across the

104 midline (three and seven days), and de novo synapse formation $(3,22)$.

105 Although a G. bimaculatus prothoracic ganglion transcriptome from this tissue was previously 106 assembled, analyzed, and mined (15), the present study re-assembled a new transcriptome based

107 on those original cleaned and trimmed RNA-Seq reads. Five individual de novo transcriptomes

108 were built in Trinity using five different k-mer lengths (21, 25, 27, 30, and 32). Transcriptome

109 construction with longer k-mer lengths produces more reliable contigs, though biased toward

110 highly expressed transcripts. In a complementary fashion, using a shorter k-mer length produces

111 a more exhaustive set of contigs though also one more prone to noise $(23,24)$. This trade-off

112 between bias and noise induced by the choice of k-mer length suggests how a higher quality de

113 novo assembly can be derived by integrating multiple k-mer lengths into an analysis (23). By

114 combining results across k-mer lengths, we ensured that a complementary selection of contigs

115 was included in the analysis $(23,25,26)$. Correspondingly, we combined the five assemblies into

116 a single reference transcriptome and subsequently filtered redundancies and fragments. 
117 The individual assemblies had an N50 ranging from 1,219 - 2,341, with the longer k-mer 118 assemblies yielding a longer N50 (Table 1). The median, average, and maximum contig length

119 also increased as the k-mer length was increased. The total number of Trinity "genes" ranged 120 from 283,278 to 351,829 , with higher k-mer assemblies yielding fewer predicted genes. The GC 121 content for each assembly remained roughly constant, between 40-41\%. The overall alignment 122 was greater than $98 \%$, with multimapping percentages between $90.04-93.33 \%$ (Table 1). This

123 high multimapping percentage can likely be attributed to the Trinity assembly process, which is 124 conservative in its separation and identification of unique transcripts, producing high intra125 assembly redundancy (27).

126 
127 Table 1: Individual k-mer assembly details.

\begin{tabular}{l|c|c|c|c|c|}
\hline & $\mathrm{k}-\mathrm{mer}=21$ & $\mathrm{k}-\mathrm{mer}=25$ & $\mathrm{k}-\mathrm{mer}=27$ & $\mathrm{k}-\mathrm{mer}=30$ & $\mathrm{k}-\mathrm{mer}=32$ \\
\hline Total \# bases assembled & $293,992,611$ & $404,116,670$ & $408,831,054$ & $406,965,539$ & $403,174,726$ \\
\hline Total \# assembled contigs & 405,638 & 438,593 & 431,712 & 415,901 & 407,158 \\
\hline Total \# Trinity "genes" & 351,829 & 302,633 & 297,584 & 288,135 & 283,278 \\
\hline Average contig length (bp) & 724.77 & 921.39 & 946 & 978.52 & 990.22 \\
\hline Median contig length (bp) & 376 & 397 & 397 & 399 & 400 \\
\hline Maximum contig & 37,575 & 44,287 & 44,328 & 44,352 & 44,331 \\
length (bp) & 1219 & 2000 & 2141 & 2272 & 2341 \\
\hline N50 (bp) & 40.94 & 40.39 & 40.32 & 40.19 & 40.14 \\
\hline GC count for & 98.61 & 98.58 & 98.66 & 98.71 & 98.74 \\
\hline assembly (\%) & 4.4 & 3.69 & 2.71 & 2.08 & 2.55 \\
\hline Overall alignment & 90.04 & 90.95 & 92.21 & 93.33 & 92.9 \\
\hline Reads mapped 1 time (\%) & & & & & \\
\hline Reads mapped & & & & & \\
\hline Time (\%) & & & & & \\
\hline
\end{tabular}

128 Table 1. Summary metrics for five different de novo transcriptomes built with five different k-mer 129 lengths.

130

131 The five transcriptomes were combined to generate a transcriptome with a total of 2,099,002

132 contigs (Figure 1), presumably many of which were redundant. We used the EvidentialGene

133 tr2aacdsmRNA classifier to filter the redundancies within our transcriptome, which were present

134 due to both intra- and inter-assembly redundancies (21). The EvidentialGene program employs

135 an algorithm that operates on the open reading frames of the contigs to generate a non-redundant

136 transcriptome containing the optimal set of transcripts based on biological relevance and coding

137 potential $(21,28)$. This program is often used in 'over-assembly' procedures where multiple

138 assemblies are combined (29-31). With our multi-k-mer assembly, EvidentialGene produced a 
139 main 'okay' set, containing 55,895 contigs, and an alternative 'okalt' set, containing 143,364

140 contigs, which were combined to produce a final transcriptome with a total of 199,357 contigs,

141 reducing the overall number of contigs by $90.5 \%$. BLAST searches across all the contigs yielded

142 matches for 127,324 transcripts, a $63.87 \%$ BLAST hit rate for the entire transcriptome. The

143 number of Trinity predicted genes after running EvidentialGene dropped slightly to 132,972 . The

144 multimapping percentage was reduced from approximately $90 \%$ to around $21 \%$.

145 To check the accuracy of the sequences predicted in the transcriptome, we used Sanger

146 sequencing to independently confirm the sequences of six randomly selected transcriptome

147 transcripts. Of these six, four of them were predicted to contain complete open reading frames

148 (ORFs), and two were missing the 3' end. We analyzed 14,299 nucleotides of 15,478 predicted

149 base pairs (92\%). The number of substitutions (16), insertions (84), and deletions (0) were noted;

150 overall, these differences accounted for approximately $\sim 0.1 \%$ of the sequenced nucleotides (data

151 not shown). A few additional randomly selected sequences were highly repetitive and were not

152 amenable to Sanger sequencing; we did not proceed with an analysis of any of these candidates.

153 Differential expression during compensatory plasticity

154 To determine genes that were differentially regulated during compensatory plasticity, the

155 reads for each of the 16 Illumina libraries, which excluded the two outliers and three backfill

156 libraries (see Methods), were mapped back to our multiple k-mer transcriptome creating a counts

157 matrix. Pairwise comparisons of normalized counts data from deafferented $v s$. control crickets

158 were performed at each time point using both algorithms, EdgeR and DESeq2 (See Supplemental

159 Materials). The distribution of differentially expressed genes was initially visualized using

160 volcano plots (Figure 2). These plots revealed slightly different distributions of upregulated

161 versus downregulated genes between the two programs. Overall, however, we saw strong 
162 correlations between these two programs for all time points (Figure 3), with the exception of a

163 few of the high fold-change candidates. Within this range, EdgeR was consistently more

164 conservative than DESeq2, which was especially true for a small number of upregulated

165 candidates (Figure 3a-c).

166 The majority of the transcripts were upregulated in the 2 to 4 -fold range at one day $(59 \%$

167 of the transcripts), three days (55\% of the transcripts), and seven days ( $45 \%$ of the transcripts).

168 The next largest group of transcripts was upregulated at 0 to 2 -fold at one day (33\% of

169 transcripts), three days ( $41 \%$ of transcripts), and seven days (39\% of transcripts). For those

170 candidates that were downregulated, a majority of them at one day $(63 \%)$ and three days $(86 \%)$

171 were downregulated less than 2-fold. At seven days, the bulk of candidates $(70 \%)$ were

172 downregulated 2 to 4-fold. Analysis of 10 of the transcripts at each time point with the largest

173 fold changes revealed that most were unidentified and did not match anything in the NCBI

174 database when BLASTed. A few of these transcripts did have BLAST hits, such as a mucin-

175 5AC-like (down at one day), larval cuticle protein-3-like (down at seven days), and hypothetical

176 accessory gland protein (up at three and seven days).

177 Using EdgeR, 261 genes were found to be downregulated at one day post-deafferentation,

1781,675 genes were downregulated at three days post-deafferentation, and 580 genes were

179 downregulated at seven days post-deafferentation (Figure 4a). Additionally, 2,234 genes were

180 determined to be upregulated at one day post-deafferentation, 1,860 genes upregulated at three

181 days post-deafferentation, and 290 genes upregulated at seven days post-deafferentation (Figure

$1824 b)$.

183 A similar pairwise comparison of deafferented versus control crickets was performed

184 using the DESeq2 software and revealed that 985 genes were downregulated at one day post- 
185 deafferentation, 3,049 genes were downregulated at three days, and 448 genes were

186 downregulated at seven days (Figure 4c). Additionally, 3,589 genes were upregulated at one day

187 post-deafferentation, 1,424 genes were upregulated at three days, and 535 genes were

188 upregulated at seven days (Figure 4d).

189 From these sets, simple comparisons were created to determine the number of genes

190 upregulated and downregulated across multiple timepoints. In EdgeR, there were four genes

191 downregulated at one and three days, two genes at one and seven days, two genes at three and

192 seven days, and 0 genes differentially downregulated across all three time points (Figure 4a). For

193 the upregulated genes, there were 174 genes differentially regulated at one and three days, 18

194 genes at one and seven days, 18 genes at three and seven days, and 32 genes at all three time

195 points (Figure 4b). Comparing the DESeq2 sets of genes across multiple timepoints showed that

196 there were nine genes downregulated at one and three days, one gene downregulated at one and

197 seven days, four genes downregulated at three and seven days, and 0 genes downregulated at all

198 three timepoints (Figure 4c). Additionally, 168 genes were found to be upregulated at one and

199 three days, 73 genes at one and seven days, 23 genes at three and seven days, and 40 genes at all

200 three time points (Figure 4d).

201 Finally, simple comparisons were performed between EdgeR and DESeq2 with genes

202 determined to be differentially expressed at each of the three times points. For downregulated

203 genes, 180 were identified at one-day post deafferentation, 1,604 at three days, and 367 at seven

204 days. For upregulated genes, 2,099 were identified at one-day post deafferentation, 1,043 at three

205 days, and 272 at seven days (Figure 5). The genes found to be differentially regulated by both

206 programs were used for further analysis. 
DESeq2 and EdgeR are the leading programs used for the analysis of RNA-Seq data,

208 with thousands of reports both using these methods for analyzing differential expression and

209 comparing their computational methods $(32,33)$. While both operate under the hypothesis that

210 the majority of genes are not differentially expressed, they employ different computational

211 methods, especially with respect to the normalization process, to determine differentially

212 expressed genes (33). EdgeR and DESeq2 both use a normalization by distribution method, but

213 EdgeR relies on the Trimmed Mean of the M-values method, whereas DESeq2 uses a Relative

214 Log Expression method (34-37). Since different methods rely on differing assumptions in order

215 to identify differentially expressed genes, the results will vary slightly. One experiment

216 comparing EdgeR and DESeq2 found relatively similar lists of differentially expressed genes

217 produced by the two programs, with EdgeR producing more conservative, smaller gene lists (32).

218 In this study we decided to use two different programs to conduct the differential expression

219 analysis in order to create a smaller, more conservative set of genes for future functional

220 analyses. Out of the six comparisons between EdgeR and DESeq2 (upregulated and

221 downregulated at one, three, and seven days post injury), four out of the six resulted in EdgeR

222 producing a smaller set of genes than DESeq2 (Figure 5), in line with the study by Raplee and

223 colleagues (32). Although the two programs generated varying numbers of differentially

224 regulated genes, similar patterns in relative numbers were observed. Both programs showed a

225 decrease in the number of genes upregulated across time. For the downregulated genes, a peak in

226 the number of differentially regulated genes was found at three days post injury. This similarity

227 was expected given the relative similarity and previous studies of both analysis programs. 


\section{BLAST and Gene Ontology Annotations}

Once we had identified a conservative set of transcripts predicted to be differentially

230 regulated, we used BLAST2GO (38) to try to identify them. Not all the transcripts inputted into

231 the BLAST2GO program resulted in BLAST hits and/or GO annotations. At one day

232 downregulated, 71\% of genes had both BLAST and GO results and an additional 10\% had only

233 BLAST hits. At three days downregulated, 36\% of genes had both BLAST and GO results and

234 an additional $6 \%$ had only BLAST hits. At seven days downregulated, $31 \%$ of genes had both

235 BLAST and GO results and an additional 6\% had only BLAST hits. For the one day upregulated,

$23653 \%$ of genes had both BLAST and GO results and an additional 10\% had only BLAST hits. At

237 three days upregulated, 59\% of genes had both BLAST and GO results and an additional 15\%

238 had only BLAST hits. At seven days upregulated, 50\% of genes had both BLAST and GO results

239 and an additional 15\% had only BLAST hits (Figure 6).

$240 \quad$ For the six lists of differentially expressed transcripts, there was a range between $37-81 \%$

241 of transcripts having BLAST hits against the nr database and 23-62\% against the manually

242 curated and annotated Swiss-Prot database. After mapping with GO terms, this was reduced to

243 about $31-59 \%$. This left close to half of the differentially regulated transcripts with no functional

244 information. These transcripts could represent uncharacterized proteins, which may or may not

245 be playing an important role in the compensatory growth response. Since we performed a

246 BLASTx looking at proteins, it is also possible that these transcripts are non-coding RNAs.

247 Although polyA selection was used as part of the RNA-Seq process, this may not be completely

248 efficient in removing all non-coding RNAs, specifically long non-coding RNAs $(39,40)$. Finally,

249 it is also possible that there were issues within these transcripts themselves, either due to an error 
250 during the assembly process or the sequences being too short to be matched with confidence.

251 Regardless, we completed no further analysis of these transcripts.

252 One interesting group of proteins that was found to be upregulated at one day was the

253 protein yellow family. Protein yellow belongs to the major royal jelly protein family and are

254 secreted proteins found in the extracellular region (41). Protein yellow was first characterized in

255 Drosophila melanogaster for its role in pigmentation (42). Other research in honeybees revealed

256 the importance of royal jelly proteins in development and social behavior in addition to a

257 possible role in the CNS $(41,43)$. However, the function of these yellow/royal jelly proteins is

258 not completely understood (42). While the role of these proteins in crickets is unclear, they were

259 statistically differentially regulated and would be an interesting molecular family to investigate

260 for their role in deafferentation-induced plasticity.

\section{GO Term Distributions}

262 Based on a preliminary grouping of GO terms by the three root classes, it appeared that

263 several classes of GO terms were found to be associated with our differentially expressed genes

264 (Figure 7). While the top five represented GO terms encompassed most of the GO terms in the

265 cellular component category, there was a much broader range of GO terms represented in the

266 molecular function and biological process categories

267 Web Gene Ontology Annotation Plot $(44,45)$ was used to plot a broader distribution of

268 GO terms and visually compare annotations among timepoints (Figure 8). Cellular component,

269 molecular function, and biological process were displayed on traditional WEGO histograms

270 (Figure 8a, b). The percentage of genes indicates the percentage of the genes within a given list

271 that were annotated with the given GO term or one of the child nodes of the term. GO terms with 
272 higher percentage representation included terms describing membrane-related components as

273 well as terms related to catalytic activity, binding, and metabolic and cellular processes.

274 Gene Ontology Categories

275 We examined whether any of the candidate GO terms we identified here were associated

276 with injury-related plasticity paradigms identified in other species. For example, perhaps

277 successful regeneration after injury depends on the recapitulation of developmental proteins that

278 promote neurogenesis (46) or guide axons and dendrites (18). If these molecular strategies were

279 important for the plasticity observed in the cricket, we would predict that we might see changes

280 in the expression of transcription factors involved in neurogenesis and/or the regulation of

281 several classes of guidance cues normally involved in midline control in insect embryos. When

282 searching our differentially regulated candidates, a few genes downregulated at three days were

283 associated with GO terms that were related to neurogenesis (GO:0007465: R7 cell fate

284 commitment and GO:0045466: R7 cell differentiation). We found only one transcript that was

285 annotated with an axon guidance-related GO term, which was identified as a "twitchin-like

286 protein," (Table 2). Twitchin/Unc-22 is a large protein kinase thought to be important in muscle

287 development and function (47).

288 An initial study of our original single k-mer transcriptome explored this developmental

289 recapitulation hypothesis by mining the transcriptome for the presence of various guidance

290 molecules (15). Though transcripts corresponding to the signaling families, slit, netrin, ephrin,

291 and semaphorin were identified within this adult transcriptome, the BLAST and Gene Ontology

292 analyses performed here did not identify an abundant number of guidance molecules as

293 differentially regulated. Despite this result, however, it is important to note that the transcriptome

294 and differential expression analysis were performed on the whole prothoracic ganglion, which 
295 could mask important changes that occur in single cells after deafferentation, such as in

296 ascending neuron 1 and 2 (AN-1 and AN-2). Single cell RNA-Seq analysis of the ANs could

297 help to determine whether there are changes in expression occurring on a smaller anatomical

298 level.

299 Based on results from different types of injury model systems in other organisms,

300 additional functional categories that we hypothesized could change during the compensatory

301 growth response were those related to apoptosis $(48,49)$ and Wnt signaling $(50)$. In our

302 differentially expressed gene sets we did not find enrichment in terms related to apoptosis.

303 Searching our results for Wnt-related GO terms, revealed a few genes annotated with Wnt

304 pathway members at 3 days post deafferentation (Table 2). These genes had a top BLAST hit of

305 atrial natriuretic peptide-converting enzyme isoform X1, Frizzled-4, and secreted frizzled-related

306 protein 5-like.

307 We looked for the presence of a number of additional groups of proteins that influence

308 neuronal morphogenesis, plasticity, or remodeling (Table 2). For example, the matrix

309 metalloproteases (MMPs) are required for axonal guidance (51) as well as dendritic remodeling

310 during metamorphosis in Drosophila melanogaster (52). Importantly, the expression of some

311 MMPs appear to contribute to poor recovery after spinal cord injury in mammals (53). We did

312 not find enrichment in any of these terms at any time point (Table 2), indicating that the injury-

313 induced anatomical plasticity in the cricket may rely on different pathways than have been

314 identified in other species. Furthermore, it is notable that factors, such as MMPs, that can restrict

315 growth or contribute to pruning in other organisms are not upregulated upon injury in the cricket.

316 Several GO terms associated with the candidates found in a previous subtraction

317 hybridization study (54) were also found to be differentially expressed in the present experiment, 
318 often showing significant changes in expression at the three- and seven-day post-deafferentation

319 time point (Table 2, bold). These include oxidoreductase, alpha-amylase, endoglucanase, and

320 alcohol dehydrogenase. As noted by Horch and colleagues (54), many of these enzymes have

321 been observed in the hemolymph of insects and play a role in metabolism and immune response.

322 Although it is possible that these findings are due to contaminants during the extraction of the

323 prothoracic ganglion, the results would imply that the extractions differed between control and

324 experimental animals in multiple experiments. Given that the differential expression of several

325 enzyme transcripts was found both in this study and in the SSH study, it is less likely that these

326 enzymes are artifact or contamination effects. Particularly, several differentially regulated

327 transcripts were associated with oxidoreductase activity across all time points. The BLAST hits

328 of these transcripts showed some of the enzymes to be retinal dehydrogenases. Retinal

329 dehydrogenase along with alcohol dehydrogenase, another regulated GO term, are involved with

330 the production of retinoic acid. Retinoic acid has been shown to be involved with development,

331 regeneration, synaptic plasticity, and neurite outgrowth (55-57) implying that regulation of

332 retinoic acid production may influence these processes. Another class of oxidoreductases that

333 appeared abundant within the BLAST hits was the cytochrome P450 family. Cytochrome P450 is

334 a superfamily of monooxygenase enzymes and several families of cytochrome P450 exist in

335 insects. These enzymes are known to have a variety of functional roles in insects including

336 growth and development (58). Cytochrome P450 has also been shown to regulate ecdysone

337 signaling in insects, including crickets $(59,60)$. Ecdysone signaling is crucial for developmental

338 processes and morphogenesis, but has also been shown to be important in the dendritic

339 remodeling of Drosophila melanogaster sensory neurons $(52,59)$. While these protein families 
340 represent some of the transcripts annotated with "oxidoreductase activity", given the wide range 341 of such transcripts, it is difficult to discern the role of all of the regulated proteins. 
343 Table 2: Evaluation of the number and fold-change of GO terms of interest.

\begin{tabular}{|c|c|c|c|c|c|c|c|}
\hline GO ID & GO Term & Down 1 & Down 3 & Down 7 & $\mathrm{Up} 1$ & Up 3 & $\mathrm{Up} 7$ \\
\hline GO:0007411 & axon guidance & N/A & N/A & N/A & N/A & $\begin{array}{c}1 \\
(3.10) \\
\end{array}$ & N/A \\
\hline GO:0048813 & $\begin{array}{l}\text { dendrite } \\
\text { morphogenesis }\end{array}$ & N/A & N/A & N/A & N/A & N/A & N/A \\
\hline GO:0022008 & neurogenesis & N/A & $\begin{array}{c}6 \\
(1.00 \pm 0.01) \\
\end{array}$ & $\mathrm{N} / \mathrm{A}$ & N/A & $\begin{array}{c}1 \\
(3.10) \\
\end{array}$ & N/A \\
\hline GO:0008219 & cell death & $\mathrm{N} / \mathrm{A}$ & $\mathrm{N} / \mathrm{A}$ & N/A & N/A & $\mathrm{N} / \mathrm{A}$ & N/A \\
\hline GO:0099544 & perisynaptic space & $\mathrm{N} / \mathrm{A}$ & N/A & N/A & N/A & $\mathrm{N} / \mathrm{A}$ & N/A \\
\hline GO:1990773 & MMP secretion & $\mathrm{N} / \mathrm{A}$ & N/A & N/A & N/A & $\mathrm{N} / \mathrm{A}$ & $\mathrm{N} / \mathrm{A}$ \\
\hline GO:0198738 & $\begin{array}{l}\text { cell-cell signaling by } \\
\text { wnt }\end{array}$ & $\mathrm{N} / \mathrm{A}$ & $\begin{array}{c}4 \\
(-1.99 \pm 0.34) \\
\end{array}$ & N/A & N/A & $\begin{array}{c}1 \\
(1.28) \\
\end{array}$ & $\begin{array}{c}1 \\
(1.20) \\
\end{array}$ \\
\hline GO:0004185 & $\begin{array}{l}\text { serine-type } \\
\text { carboxypeptidase } \\
\text { activity }\end{array}$ & N/A & N/A & N/A & N/A & N/A & N/A \\
\hline GO:0003735 & ribosomal constituent & N/A & N/A & N/A & N/A & $\mathrm{N} / \mathrm{A}$ & N/A \\
\hline GO:0003743 & $\begin{array}{l}\text { translation initiation } \\
\text { factor activity }\end{array}$ & N/A & N/A & N/A & N/A & N/A & N/A \\
\hline GO:0008135 & $\begin{array}{l}\text { translation factor } \\
\text { activity, RNA binding }\end{array}$ & N/A & N/A & $\mathrm{N} / \mathrm{A}$ & N/A & N/A & $\begin{array}{c}12 \\
(2.85 \pm 0.24) \\
\end{array}$ \\
\hline GO:0008106 & \begin{tabular}{|l|} 
alcohol \\
dehydrogenase \\
$($ NADP+) activity
\end{tabular} & N/A & N/A & $\mathrm{N} / \mathrm{A}$ & $\begin{array}{c}3 \\
(1.26+/-0.01) \\
\end{array}$ & N/A & N/A \\
\hline$G O: 0004556$ & $\begin{array}{l}\text { alpha-amylase } \\
\text { activity }\end{array}$ & N/A & N/A & $\begin{array}{c}8 \\
(-2.77 \pm 0.27)\end{array}$ & N/A & $\begin{array}{c}13 \\
(2.65 \pm 0.61)\end{array}$ & N/A \\
\hline GO:0015927 & trehalase activity & N/A & $\mathrm{N} / \mathrm{A}$ & N/A & $\mathrm{N} / \mathrm{A}$ & $\mathrm{N} / \mathrm{A}$ & $\mathrm{N} / \mathrm{A}$ \\
\hline GO:0016491 & \begin{tabular}{|l} 
oxidoreductase \\
activity
\end{tabular} & $\begin{array}{c}4 \\
(-2.38 \pm 2.24) \\
\end{array}$ & $\begin{array}{c}112 \\
(-1.40 \pm 0.43) \\
\end{array}$ & $\begin{array}{c}14 \\
(-2.82 \pm 0.45) \\
\end{array}$ & $\begin{array}{c}175 \\
(2.45+0.92) \\
\end{array}$ & $\begin{array}{c}124 \\
(2.38 \pm 0.90) \\
\end{array}$ & $\begin{array}{c}7 \\
(3.48+1.69) \\
\end{array}$ \\
\hline GO:0008810 & $\begin{array}{l}\text { cellulase activity } \\
\text { (endoglucanase) }\end{array}$ & N/A & N/A & $\begin{array}{c}3 \\
(-2.43+/-0.30)\end{array}$ & N/A & $\begin{array}{c}1 \\
(1.97) \\
\end{array}$ & N/A \\
\hline GO:0003796 & lysozyme activity & N/A & N/A & N/A & N/A & $\mathrm{N} / \mathrm{A}$ & N/A \\
\hline GO:0004129 & $\begin{array}{l}\text { cytochrome-c oxidase } \\
\text { activity }\end{array}$ & $\mathrm{N} / \mathrm{A}$ & N/A & N/A & $\mathrm{N} / \mathrm{A}$ & N/A & N/A \\
\hline GO:0016567 & protein ubiquitination & $\begin{array}{c}1 \\
(-1.60) \\
\end{array}$ & $\begin{array}{c}1 \\
(-1.24) \\
\end{array}$ & N/A & $\begin{array}{c}3 \\
(2.53 \pm 0.29) \\
\end{array}$ & $\begin{array}{c}11 \\
(2.15+0.76) \\
\end{array}$ & $\begin{array}{c}22 \\
(1.91+0.10)\end{array}$ \\
\hline GO:0019783 & $\begin{array}{l}\text { ubiquitin-like protein } \\
\text { specific protease } \\
\text { activity }\end{array}$ & N/A & N/A & N/A & N/A & N/A & N/A \\
\hline
\end{tabular}

344 Table 2: Number of transcripts associated with the selected GO terms, including any child term of the

345 GO term, at each time point. For each represented GO term, the average $+/$ - standard deviation of the

$346 \log 2$ foldchange across the transcripts is given in parentheses. GO Terms in bold indicate significant

347 changes in expression. GO IDs in italics were selected because similar transcripts were present in a prior

348 SSH study (54). 


\section{Gene Ontology Enrichment Analysis}

Metascape (61) was used to determine enriched GO terms across the differentially

353 expressed gene lists. Differentially expressed genes were first reBLASTed against the curated

354 Swiss-Prot database to retrieve appropriate gene identifiers. Similar ratios of BLAST hit

355 percentages across timepoints were observed against Swiss-Prot as with the nr database,

356 however, the percentage of genes with BLAST hits was lower when BLASTed against Swiss-

357 Prot versus the nr database (Table 3).

358 Table 3: Comparison of the percentage of genes with BLAST hits in the nr database vs.

359 Swiss-Prot.

\begin{tabular}{|c|c|c|}
\hline & $\mathrm{nr}$ & Swiss-Prot \\
\hline Down 1 & $81 \%$ & $59 \%$ \\
\hline Down 3 & $42 \%$ & $36 \%$ \\
\hline Down 7 & $37 \%$ & $23 \%$ \\
\hline Up 1 & $62 \%$ & $59 \%$ \\
\hline Up 3 & $73 \%$ & $62 \%$ \\
\hline Up 7 & $65 \%$ & $57 \%$ \\
\hline
\end{tabular}

Enrichment analysis by Metascape showed the most enriched terms at three days post-

362 deafferentation across both upregulated and downregulated genes. Examining the multi-list

363 analysis, there were 22 enriched GO terms found including those related to morphogenesis,

364 extracellular space, and neuron fate commitment (Figure 9). No enriched GO terms were found

365 in the upregulated gene set at seven days post-deafferentation. 
One category of interest that was revealed in this enrichment analysis was the GO Term

367 "Regulation of Toll-signaling pathway." Toll receptors are most commonly associated with their

368 function in immunity and development, however, research in Drosophila melanogaster suggests

369 that they may also play a role in regulating cell number, connectivity, and synaptogenesis (62).

370 Activation of Toll receptors can regulate cell number through either neuroprotective and cell

371 survival or pro-apoptotic functions, depending on the receptor type. These functions of Toll

372 receptors were shown to exist in both development and adulthood (63). Toll receptors,

373 specifically Toll-6 and Toll-7, have also been shown to have neurotrophic receptor-like functions

374 through their ability to bind multiple ligands, including neurotrophin-like proteins in

375 invertebrates (64). Neurotrophins are known to regulate cell proliferation and neuronal survival

376 and development, thereby suggesting an important role for Toll receptors in neuronal systems

377 (63,64). Furthermore, in Drosophila melanogaster the receptor Toll-8 was shown to positively

378 regulate synaptic growth through a retrograde neurotrophin-like signaling mechanism (65).

379 These studies show that Toll signaling may play an important role in regulating structural

380 plasticity in invertebrates and, given their enrichment in our differentially regulated gene set,

381 may be crucial to the dendritic reorganization observed in the cricket.

\section{Conclusions}

384 Unilateral tympanal organ removal in the cricket, Gryllus bimaculatus, leads to a robust

385 reorganization of dendrites in the auditory system of the prothoracic ganglion. This novel growth 386 and de novo synapse formation restores the ability of the deafferented neurons to respond to

387 sound. Our transcriptomic analyses identified thousands of transcripts up- and down-regulated

388 after deafferentation. We highlight transcriptional changes related to protein translation and 
degradation, enzymatic activity, and Toll signaling that appear to be enriched after

390 deafferentation. The data presented here allows the development of targeted hypotheses that

391 could elucidate the mechanisms responsible for the deafferentation-induced synaptic plasticity in

392 the auditory system of crickets. The mechanisms at play here can be compared and contrasted

393 with those identified in the terminal ganglion of the cricket after unilateral loss of a cercus.

395 Methods

Animals, injury, and library preparation

Prothoracic ganglia from approximately 60 adult, male Mediterranean field crickets,

398 Gryllus bimaculatus were harvested and 21 individual ganglia were ultimately used as the

399 sources of RNA for this transcriptome (15). Male crickets that were adults for 3-5 days received

400 either a control amputation of the distal segment of the left tarsus ("foot chop" control crickets),

401 or the left prothoracic leg was severed mid-femur removing the auditory organ and deafferenting

402 the ipsilateral central auditory neurons ("deafferented" experimental crickets). Males were

403 chosen due to the potential sexual dimorphism in rates of dendritic growth after deafferentation

404 (22). (15). Several crickets were prepared for backfill as previously described (15). Prothoracic

405 ganglia were removed from these crickets 1, 3, or 7 days after amputation at the femur or tarsus

406 and total RNA was purified as previously described.

The QIAGEN RNeasy Lipid Tissue Minikit was used to purify total RNA from each

408 sample individually. RNA concentrations were assessed after TURBO DNA-free treatment

409 (Ambion by Life Technologies) with a nanospectrophotometer (Nanodrop, Thermo Fisher

410 Scientific) or a fluorometer (Qubit, Thermo Fisher Scientific). An Agilent 2100 Bioanalyzer

411 (Applied Biosystems, Carlsbad, CA) was used to further assess sample quality. Based on 
412 evaluation of RNA quality and concentration of individual ganglion samples, the best 3 samples

413 for each condition were selected for sequencing. Standard Illumina paired-end library protocols

414 were used to prepare samples. The Illumina Hiseq 2500 platform, running v4 chemistry to

415 generate $\sim 25 \mathrm{M}$ paired end reads of $100 \mathrm{bp}$ in length for each sample, was used to sequence the

416 RNA (15).

\section{Transcriptome assembly}

418 Trinity software (Trinity-v2.6.5) was run using previously processed and filtered reads of

419 prothoracic ganglion libraries (15). A multi-k-mer assembly was created by building five de novo

420 transcriptomes using a single k-mer length $(21,25,27,30,32)$ and subsequently combining

421 them. The following parameters were used: minimum contig length of 200, library normalization

422 with maximum read coverage 50, RF strand specific read orientation, maximum memory,

$423250 \mathrm{~GB}$, and 32 CPUs. Individual assemblies were analyzed using the TrinityStats.pl script and

424 alignment statistics were obtained using Bowtie2 (v 2.3.4.1). The PRINSEQ interactive program

425 (66) was used to generate additional summary statistics on each assembly.

426 A k-mer number identity was added to each contig's Trinity ID, all five assemblies were

427 concatenated, and the Evidential Gene program was used to create a single non-redundant

428 assembly. Evidential Gene relies on the Transdecoder.LongOrfs method, identifies the longest

429 ORFs, removes fragments, and uses a BLAST on self to identify highly similar sequences (98\%).

430 The main (okay) and alternative (okalt) sets output from Evidential Gene were combined into a

431 final FASTA file used as the transcriptome for all subsequent analyses. Bam files, sorted bam

432 files, bam index files, and idxstats.txt files were created using samtools (67). This assembly is

433 publicly available at NCBI (Bioproject: PRJNa376023, SUB8325660). The metajinomics python

434 mapping tools (68) were used to generate a counts matrix. 


\section{Coverage Analysis}

Samtools was used to extract the sequencing depth at every base position for each contig

437 in every cricket sample, and a python script was used to extract the mean and standard deviation

438 of depth for each contig. The package plotly in R (69) was used to plot the depth of each cricket

439 sample. Graphs were visually compared to determine outliers. Two outliers, 1C1 and 7C2, were

440 removed.

441 EdgeR and DESeq were used to run the differential expression analysis, $(35,37)$. The

442 raw read counts generated for each of the libraries, excluding the outliers and the backfill

443 conditions, were used as input to both programs. Similar filtering and normalization functions

444 were used in both programs to exclude any contigs that did not have at least one count per

445 million in at least two libraries. Comparisons between control and deafferented samples were

446 performed at each time point to create lists of upregulated and downregulated genes with a $\mathrm{p}$

447 value cutoff of 0.05 . Pairwise comparison results were then compared across time points and

448 were then compared and visualized between the two programs. Another set of lists containing the

449 genes overlapping the two programs was created for continued analysis. The EnhancedVolcano

450 package available in $\mathrm{R}$ was used to visualize differential gene expression in volcano plots (70).

$451 \quad$ PCR Confirmation

452 Six sequences were randomly selected for amplification and Sanger sequencing in order

453 to validate the assembly. Sets of primers were designed to obtain the sequence of most of each

454 sequence as predicted by the Trinity assembly. Primers, available on request, were designed for

455 TRINITY21_DN57089_c8_g2_i1.p1 (hypothetical coiled-coil domain protein),

456 TRINITY21_DN58301_c9_g1_i8.p1 (eukaryotic translation initiation factor 4 gamma 3-like),

457 TRINITY25_DN131062_c0_g1_i1.p1 (protein unc-13 homolog 4B), 
TRINITY27_DN140563_c0_g1_i5.p1 (syntaxin-binding protein 5),

459 TRINITY32_DN141398_c1_g1_i7.p1 (cytochrome P450 301a1),

460 TRINITY21_DN54942_c12_g1_i5.p1 (kinesin light chain). cDNA derived from RNA purified

461 form independent control and deafferented prothoracic ganglion samples was used for PCR. PCR

462 amplicons were gel purified and sequenced, and sequences were aligned and analyzed in

463 Geneious Prime Software (Version 2019.2.3).

464

465

466

467

468

469

470 August 2, 2018.

\section{1}

472

473

474

475

\section{BLAST Searches}

A Perl script was used to extract differentially expressed sequences. The NCBI BLASTx local tool (71) was used to identify proteins similar to the translated nucleotide query sequences. An E-value cutoff of 1e-3 was used and max target sequences was set to 1, and max hits per sequence was set to 1, resulting in the output of only the top hit. Query sequences were BLASTed against the entire non-redundant database downloaded from the NCBI website on

\section{Gene Ontology Analysis}

The program, BLAST2GO provided GO annotations for differentially regulated genes (38) using the following parameters: BLASTx-fast against the nr database, number of blast BLAST hits $=20$, E-value of 1.0 e -3 , word size of 6 , hsp length cutoff of 33, with default mapping and annotation settings. GO terms found to be associated with various genes were manually grouped according to GO subtype (cellular component, biological process, or molecular function) and plotted to view the distribution across time points. The web-based CateGOrizer program was used to batch analyze each set of GO terms and determine the number of GO terms under higher order GO classes of interest (72). WEGO 2.0 (Web Gene Ontology Annotation Plot) with a GO level of 2 was also used to plot histograms of the GO annotations for 
481 the differentially regulated genes (45). To further analyze the differentially expressed genes, an

482 enrichment analysis was performed with Metascape (61). A multiple gene list analysis looking at

483 the enrichment of the three classes of Gene Ontology terms was performed using Drosophila

484 melanogaster as the analysis species.

485

\section{Availability of data and material}

487 Initial description of assembly of this transcriptome in Fisher et al., 2018. Re-assembly was 488 completed as described above and is publicly available at NCBI (Bioproject: PRJNa376023,

489 SUB8325660)

490

491 Availability of supporting data

492 Supporting data are included as Supplemental Materials and on NCBI.

493

494 Additional files:

495 Files listing all up- and downregulated genes found to be significant at or below $\mathrm{p}=0.05$ by both 496 DESeq2 and EdgeR, by just DESeq2 (DESonly), or by just EdgeR (EDRonly) are included in 497 Supplemental Materials.

499 Ethics approval and consent to participate

500 All experimental protocols were approved by Bowdoin College's IACUC committee, and all 501 experimental methods were carried out in accordance with approved guidelines and regulations.

502 Experimental design and methods reporting were carried out in compliance with ARRIVE 503 guidelines. 


\section{Consent for publication}

$505 \quad \mathrm{~N} / \mathrm{A}$

506

507 Competing interests

508 The authors declare that they have no competing interests

509

$510 \quad$ Funding

511 Research reported in this project was supported by an Institutional Development Award (IDeA)

512 from the National Institute of General Medical Sciences of the National Institutes of Health

513 under grant number P20GM103423.

514

\section{Authors' contributions}

$516 \mathrm{HF}$, assisted by LL, collected tissue and HF completed the original transcriptome assembly; FW

517 and MM reassembled using multiple K-mers, compacted the new transcriptome using Evigene

518 and did the differential expression analysis; JB, JJJ, LSMP, and TAM completed the sanger

519 sequencing analysis; FW wrote the paper; JO and SK consulted on the statistical differential

520 expression analysis; HWH obtained funding for this project and contributed to the writing. All

521 authors read and approved the final manuscript

522

\section{Acknowledgements}

524 We thank Marko Melendy for animal care support. 


\section{References}

527 1. Prigge CL, Kay JN. Dendrite morphogenesis from birth to adulthood. Current Opinion in $528 \quad$ Neurobiology. 2018 Dec 1;53:139-45.

529 2. Sampaio-Baptista C, Sanders Z-B, Johansen-Berg H. Structural Plasticity in a with motor $530 \quad$ learning and stroke rehabilitation. Annual Review of Neuroscience. 2018;41(1):25-40.

3. Brodfuehrer PD, Hoy RR. Effect of auditory deafferentation on the synaptic connectivity of

4. Horch HW, Sheldon E, Cutting CC, Williams CR, Riker DM, Peckler HR, et al. Bilateral consequences of chronic unilateral deafferentation in the auditory system of the cricket Gryllus bimaculatus. Dev Neurosci. 2011;33(1):21-37.

5. Schmitz B. Neuroplasticity and phonotaxis in monaural adult female crickets (Gryllus bimaculatus de Geer). Journal of Comparative Physiology A: Neuroethology. 1989;164(3):343-58.

6. Popov AV, Markovich AM, Andjan AS. Auditory interneurons in the prothoracic ganglion

7. Poulet JFA, Hedwig B. Tympanic membrane oscillations and auditory receptor activity in the stridulating cricket Gryllus bimaculatus. Journal of Experimental Biology. 2001;204(7):1281-93.

8. Wohlers DW, Huber F. Topographical organization of the auditory pathway within the prothoracic ganglion of the cricket Gryllus campestris L. Cell Tissue Res. 1985;239(3):555-65.

9. Hoy RR, Nolen TG, Casaday GC. Dendritic sprouting and compensatory synaptogenesis in an identified interneuron following auditory deprivation in a cricket. Proc Natl Acad Sci USA. $1985 ; 82(22): 7772-6$.

10. Schildberger K, Wohlers DW, Schmitz B. Morphological and physiological changes in central auditory neurons following unilateral foreleg amputation in larval crickets. Journal of Comparative Neurology. 1986;158:291-300.

11. Ylla G, Nakamura T, Itoh T, Kajitani R, Toyoda A, Tomonari S, et al. Cricket genomes: the genomes of future food [Internet]. Genomics; $2020 \mathrm{Jul}$ [cited $2020 \mathrm{Aug} 6$ ]. Available from: http://biorxiv.org/lookup/doi/10.1101/2020.07.07.191841

12. Bando T, Ishimaru Y, Kida T, Hamada Y, Matsuoka Y, Nakamura T, et al. Analysis of RNA-Seq data reveals involvement of JAK/STAT signaling during leg regeneration in the cricket Gryllus bimaculatus. Development. 2013;140(5):959-64.

13. Zeng V, Extavour CG. ASGARD: an open-access database of annotated transcriptomes for emerging model arthropod species. Database (Oxford). 2012;2012:bas048. 
14. Zeng V, Ewen-Campen B, Horch HW, Roth S, Mito T, Extavour CG. Developmental gene discovery in a hemimetabolous insect: De novo assembly and annotation of a transcriptome for the cricket Gryllus bimaculatus. Dearden PK, editor. PLoS ONE. 2013;8(5):e61479.

15. Fisher HP, Pascual MG, Jimenez SI, Michaelson DA, Joncas CT, Quenzer ED, et al. De novo assembly of a transcriptome for the cricket Gryllus bimaculatus prothoracic ganglion: An invertebrate model for investigating adult central nervous system compensatory plasticity. PLoS ONE. 2018;13(7):e0199070.

16. Dickson BJ. Molecular mechanisms of axon guidance. Science. 2002 Dec 6;298(5600):1959-64.

17. Tessier-Lavigne M, Goodman CS. The Molecular Biology of Axon Guidance. Science. 1996 Nov 15;274(5290):1123-33.

18. Harel NY, Strittmatter SM. Can regenerating axons recapitulate developmental guidance during recovery from spinal cord injury? Nat Rev Neurosci. 2006;7(8):603-16.

19. Kikuchi K, Kishino A, Konishi O, Kumagai K, Hosotani N, Saji I, et al. In vitro and in vivo characterization of a novel semaphorin 3A inhibitor, SM-216289 or xanthofulvin. J Biol Chem. 2003;278(44):42985-91.

20. $\mathrm{Yu} \mathrm{H-H,} \mathrm{Araj} \mathrm{HH,} \mathrm{Ralls} \mathrm{SA,} \mathrm{Kolodkin} \mathrm{AL.} \mathrm{The} \mathrm{transmembrane} \mathrm{semaphorin} \mathrm{Sema} \mathrm{I} \mathrm{is}$ required in Drosophila for embryonic motor and CNS axon guidance. Neuron. 1998;20:207-20.

21. Gilbert D. EvidentialGene: mRNA Transcript Assembly Software [Internet]. EvidentialGene: tr2aacds, mRNA Transcript Assembly Software. 2013 [cited 2020 Jan 8]. Available from: http://arthropods.eugenes.org/EvidentialGene/trassembly.html

22. Pfister A, Johnson A, Ellers O, Horch HW. Quantification of dendritic and axonal growth after injury to the auditory system of the adult cricket Gryllus bimaculatus. Front Physiol. 2013;3:367.

23. Surget-Groba Y, Montoya-Burgos JI. Optimization of de novo transcriptome assembly from next-generation sequencing data. Genome Res. 2010 Oct 1;20(10):1432-40.

24. Zhao Q-Y, Wang Y, Kong Y-M, Luo D, Li X, Hao P. Optimizing de novo transcriptome assembly from short-read RNA-Seq data: a comparative study. BMC Bioinformatics. 2011;12(Suppl 14):S2.

25. Haznedaroglu BZ, Reeves D, Rismani-Yazdi H, Peccia J. Optimization of de novo transcriptome assembly from high-throughput short read sequencing data improves functional annotation for non-model organisms. BMC Bioinformatics. 2012;13(1):170.

26. Mamrot J, Legaie R, Ellery SJ, Wilson T, Seemann T, Powell DR, et al. De novo transcriptome assembly for the spiny mouse (Acomys cahirinus). Sci Rep. 2017 Dec;7(1):8996. 
27. Cerveau N, Jackson DJ. Combining independent de novo assemblies optimizes the coding transcriptome for nonconventional model eukaryotic organisms. BMC Bioinformatics. 2016 Dec;17(1):525.

28. Gilbert DG. Longest protein, longest transcript or most expression, for accurate gene reconstruction of transcriptomes? [Internet]. Bioinformatics; 2019 Nov [cited 2020 Jan 9]. Available from: http://biorxiv.org/lookup/doi/10.1101/829184

29. Gan HM, Austin C, Linton S. Transcriptome-guided identification of carbohydrate active enzymes (CAZy) from the Christmas Island Red Crab, Gecarcoidea natalis and a vote for the inclusion of transcriptome-derived crustacean CAZys in comparative studies. Mar Biotechnol. 2018;20(5):654-65.

30. Richardson MF, Sequeira F, Selechnik D, Carneiro M, Vallinoto M, Reid JG, et al. Improving amphibian genomic resources: a multitissue reference transcriptome of an iconic invader. GigaScience [Internet]. 2018 Jan 1 [cited 2020 Jan 9];7(1). Available from: https:/academic.oup.com/gigascience/article/doi/10.1093/gigascience/gix114/4662864

31. Rivera-García L, Rivera-Vicéns RE, Veglia AJ, Schizas NV. De novo transcriptome assembly of the digitate morphotype of Briareum asbestinum (Octocorallia: Alcyonacea) from the southwest shelf of Puerto Rico. Marine Genomics. 2019;47:100676.

32. Raplee ID, Evsikov AV, Marín de Evsikova C. Aligning the aligners: Comparison of RNA sequencing data alignment and gene expression quantification tools for clinical breast cancer research. Journal of Personalized Medicine. 2019;9(2):18.

33. Varet H, Brillet-Guéguen L, Coppée J-Y, Dillies M-A. SARTools: A DESeq2- and EdgeRbased R pipeline for comprehensive differential analysis of RNA-seq data. PLOS ONE. 2016 Jun 9;11(6):e0157022.

34. Evans C, Hardin J, Stoebel DM. Selecting between-sample RNA-Seq normalization methods from the perspective of their assumptions. Briefings in Bioinformatics. 2018 Sep 28;19(5):776-92.

35. Love MI, Huber W, Anders S. Moderated estimation of fold change and dispersion for RNA-seq data with DESeq2. Genome Biol. 2014;15(12):550.

36. Maza E, Frasse P, Senin P, Bouzayen M, Zouine M. Comparison of normalization methods for differential gene expression analysis in RNA-Seq experiments. Communicative \& Integrative Biology. 2013;6(6):e25849.

37. Robinson MD, McCarthy DJ, Smyth GK. EdgeR: a Bioconductor package for differential expression analysis of digital gene expression data. Bioinformatics. 2010;26(1):139-40.

38. Gotz S, Garcia-Gomez JM, Terol J, Williams TD, Nagaraj SH, Nueda MJ, et al. Highthroughput functional annotation and data mining with the Blast2GO suite. Nucleic Acids Research. 2008;36(10):3420-35. 
39. Doughty T, Kerkhoven E. Extracting novel hypotheses and findings from RNA-seq data. FEMS Yeast Res [Internet]. 2020 Mar 1 [cited 2020 Jun 11];20(2). Available from: http://academic.oup.com/femsyr/article/20/2/foaa007/5721245

40. Zhao S, Zhang Y, Gamini R, Zhang B, von Schack D. Evaluation of two main RNA-seq approaches for gene quantification in clinical RNA sequencing: polyA+ selection versus rRNA depletion. Scientific Reports. 2018 Mar 19;8(1):4781.

41. Kucharski R, Maleszka R, Hayward DC, Ball EE. A royal jelly protein Is expressed in a subset of Kenyon Cells in the mushroom bodies of the honey bee brain. Naturwissenschaften. 1998;85(7):343-6.

42. Ferguson LC, Green J, Surridge A, Jiggins CD. Evolution of the insect yellow gene family. Molecular Biology and Evolution. 2011;28(1):257-72.

43. Albert $\breve{\mathrm{S}}$, Klaudiny J. The MRJP/YELLOW protein family of $<\mathrm{i}>$ Apis mellifera $<\mathrm{i} / \mathrm{i}>$ Identification of new members in the EST library. Journal of Insect Physiology. 2004;50(1):51-9.

44. Ye J, Fang L, Zheng H, Zhang Y, Chen J, Zhang Z, et al. WEGO: a web tool for plotting GO annotations. Nucleic Acids Research. 2006;34(Web Server):W293-7.

45. Ye J, Zhang Y, Cui H, Liu J, Wu Y, Cheng Y, et al. WEGO 2.0: a web tool for analyzing and plotting GO annotations, 2018 update. Nucleic Acids Research. 2018;46(W1):W71-5.

46. Quadrato G, Di Giovanni S. Waking up the sleepers: Shared transcriptional pathways in axonal regeneration and neurogenesis. Cell Mol Life Sci. 2013;70(6):993-1007.

47. Matsunaga Y, Qadota H, Furukawa M, Choe H (Helen), Benian GM. Twitchin kinase interacts with MAPKAP kinase 2 in Caenorhabditis elegans striated muscle. Mol Biol Cell. 2015;26(11):2096-111.

48. Baldi A, Calia E, Ciampini A, Riccio M, Vetuschi A, Persico A, et al. Deafferentationinduced apoptosis of neurons in thalamic somatosensory nuclei of the newborn rat: critical period and rescue from cell death by peripherally applied neurotrophins. Eur J Neurosci. 2000;12:2281-90.

49. Garcia-Valenzuela E, Gorczyca W, Darzynkiewicz Z, Sharma SC. Apoptosis in adult retinal ganglion cells after axotomy. J Neurobiol. 1994 Apr;25(4):431-8.

50. Herman PE, Papatheodorou A, Bryant SA, Waterbury CKM, Herdy JR, Arcese AA, et al. Highly conserved molecular pathways, including Wnt signaling, promote functional recovery from spinal cord injury in lampreys. Sci Rep. 2018;8(1):18.

51. Miller CM, Page-McCaw A, Broihier HT. Matrix metalloproteinases promote motor axon fasciculation in the Drosophila embryo. Development. 2008 Jan 1;135(1):95-109. 
52. Kuo CT, Jan LY, Jan YN. Dendrite-specific remodeling of Drosophila sensory neurons requires matrix metalloproteases, ubiquitin-proteasome, and ecdysone signaling. Proc Natl Acad Sci USA. 2005;102(42):15230-5.

53. Zhang H, Chang M, Hansen CN, Basso DM, Noble-Haeusslein LJ. Role of matrix metalloproteinases and therapeutic benefits of their inhibition in spinal cord injury. Neurotherapeutics. 2011;8(2):206-20.

54. Horch HW, McCarthy SS, Johansen SL, Harris JM. Differential gene expression during compensatory sprouting of dendrites in the auditory system of the cricket Gryllus bimaculatus. Insect Molecular Biology. 2009;18(4):483-96.

55. Aoto J, Nam CI, Poon MM, Ting P, Chen L. Synaptic signaling by all-trans retinoic acid in homeostatic synaptic plasticity. Neuron. 2008;60(2):308-20.

56. Dmetrichuk JM, Carlone RL, Spencer GE. Retinoic acid induces neurite outgrowth and growth cone turning in invertebrate neurons. Dev Biol. 2006;294:39-49.

57. Maden M. Retinoic acid in the development, regeneration and maintenance of the nervous system. Nature Reviews Neuroscience. 2007;8(10):755-65.

58. Scott JG, Wen Z. Cytochromes P450 of insects: the tip of the iceberg. Pest Management Science. 2001;57(10):958-67.

59. Chavez VM, Marques G, Delbecque JP, Kobayashi K, Hollingsworth M, Burr J, et al. The Drosophila disembodied gene controls late embryonic morphogenesis and codes for a cytochrome P450 enzyme that regulates embryonic ecdysone levels. Development. 2000;127(19):4115-26.

60. Liebrich W (Universitat U, Durnberger BB, Hoffmann KH. Ecdysone 20-monooxygenase in a cricket (Gryllus bimaculatus ensifera, Gryllidae): activity throughout adult life cycle. Comparative biochemistry and physiology : A [Internet]. 1991 [cited 2021 Feb 5]; Available from: https://agris.fao.org/agris-search/search.do?recordID=US9150408

61. Zhou Y, Zhou B, Pache L, Chang M, Khodabakhshi AH, Tanaseichuk O, et al. Metascape provides a biologist-oriented resource for the analysis of systems-level datasets. Nature Communications. 2019;10(1):1523.

62. Anthoney N, Foldi I, Hidalgo A. Toll and Toll-like receptor signalling in development. Development [Internet]. 2018 [cited 2020 Apr 17];145(9). Available from: https://dev.biologists.org/content/145/9/dev156018

63. Li G, Forero MG, Wentzell JS, Durmus I, Wolf R, Anthoney NC, et al. A Toll-receptor map underlies structural brain plasticity. Bellen HJ, Banerjee U, editors. eLife. $2020 \mathrm{Feb}$ 18;9:e52743. 
64. McIlroy G, Foldi I, Aurikko J, Wentzell JS, Lim MA, Fenton JC, et al. Toll-6 and Toll-7 function as neurotrophin receptors in the Drosophila melanogaster CNS. Nature Neuroscience. 2013;16(9):1248-56.

65. Ballard SL, Miller DL, Ganetzky B. Retrograde neurotrophin signaling through Tollo regulates synaptic growth in Drosophila. J Cell Biol. 2014 Mar 31;204(7):1157-72.

66. Schmieder R, Edwards R. Quality control and preprocessing of metagenomic datasets. Bioinformatics. 2011 Mar 15;27(6):863-4.

67. Li H, Handsaker B, Wysoker A, Fennell T, Ruan J, Homer N, et al. The sequence alignment/map format and SAMtools. Bioinformatics. 2009;25(16):2078-9.

68. Choi J. Metajinomics mapping tool. 2017; Available from: https://github.com/metajinomics/mapping_tools

69. Sievert C. Interactive Web-Based Data Visualization with R, plotly, and shiny [Internet]. Chapman and Hall/CRC; 2020. Available from: https://plotly-r.com

70. Blighe K, Rana S, Lewis M. EnhancedVolcano: Publication-ready volcano plots with enhanced colouring and labeling. [Internet]. 2020 [cited 2020 Jan 7]. Available from: https://github.com/kevinblighe/EnhancedVolcano

71. Altschul SF, Gish W, Miller W, Myers EW, Lipman DJ. Basic local alignment search tool. Journal of Molecular Biology. 1990;215(3):403-10.

72. $\mathrm{Hu} \mathrm{Z}$, Bao J, Reecy J. CateGOrizer: A web-based program to batch analyze gene ontology classification categories. Online Journal of Bioinformatics. 2008;9:108-12. 


\section{Figure legends}

725 Figure 1. Summary of workflow for multi k-mer assembly.

726

727

728

729

730

731

732

733

734

735

736

737

738

739

740

741

742

743

744

745

746

747

748

749

750

751

752

753

754

755

756

757

758

759

760

761

762

763
Figure 2. Volcano plot of differential gene expression in G. bimaculatus prothoracic ganglia at three different time points after deafferentation. The horizontal dotted line marks a p-value of 0.05 , and the vertical dotted line marks no predicted fold change. Each point represents a contig determined to be differentially regulated by EdgeR or DESeq2. Blue points represent the contigs determined to be significantly regulated.

Figure 3: Correlation of fold-changes predicted by EdgeR and DESeq2 for upregulated transcripts (left) and downregulated transcripts (right) at one, three, and seven days.

Figure 4. Differentially regulated genes across three time points. Similar patterns in relative numbers of differentially regulated genes are observed between the two programs. a) EdgeR identified downregulated genes b) EdgeR identified upregulated genes c) DESeq2 identified downregulated genes d) DESeq2 identified upregulated genes.

Figure 5. Differentially regulated genes compared across the two analytical programs, DESeq2 and EdgeR. The number of genes found to be differentially regulated by both programs varies by condition.

Figure 6. Percentage of sequences with no BLAST hits, BLAST hits, and BLAST hits with additional GO term mapping. Distribution of sequences varies across times points and regulation.

Figure 7. GO term analysis organized into the three root classes: cellular component (CC), molecular function (MF), and biological process (BP). The top 5 represented GO terms across all time points in each class are represented. Many highly represented GO terms were found in the $\mathrm{CC}$ class whereas a broader range of $\mathrm{GO}$ terms were found in the MF and $\mathrm{BP}$ classes.

Figure 8. WEGO histograms with the distribution of Gene Ontology terms grouped by cellular component, molecular function, or biological process. a) GO terms associated with downregulated genes across all time points. b) GO terms associated with upregulated genes across all time points. Percentages are noted on the left and the number of genes within the given list that were annotated with the GO term/child term are noted on the right. On the right axis, the top numbers (olive) corresponds to the one-day data, the middle numbers, (magenta) corresponds to the three-day data, and the bottom numbers correspond to the seven-day data (teal). 
764 Figure 9. Heatmap of enrichment terms as determined by Metascape. Colored by p-value as 765 indicated at the top.

766 
Figures

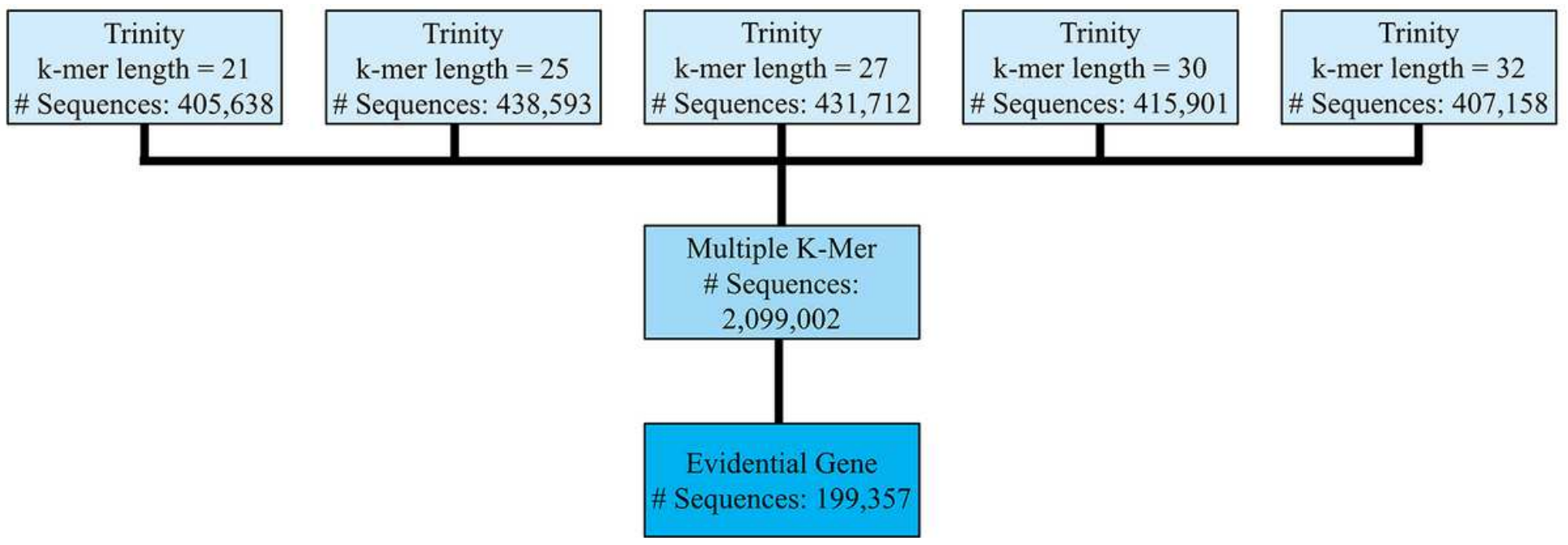

Figure 1

Summary of workflow for multi k-mer assembly. 

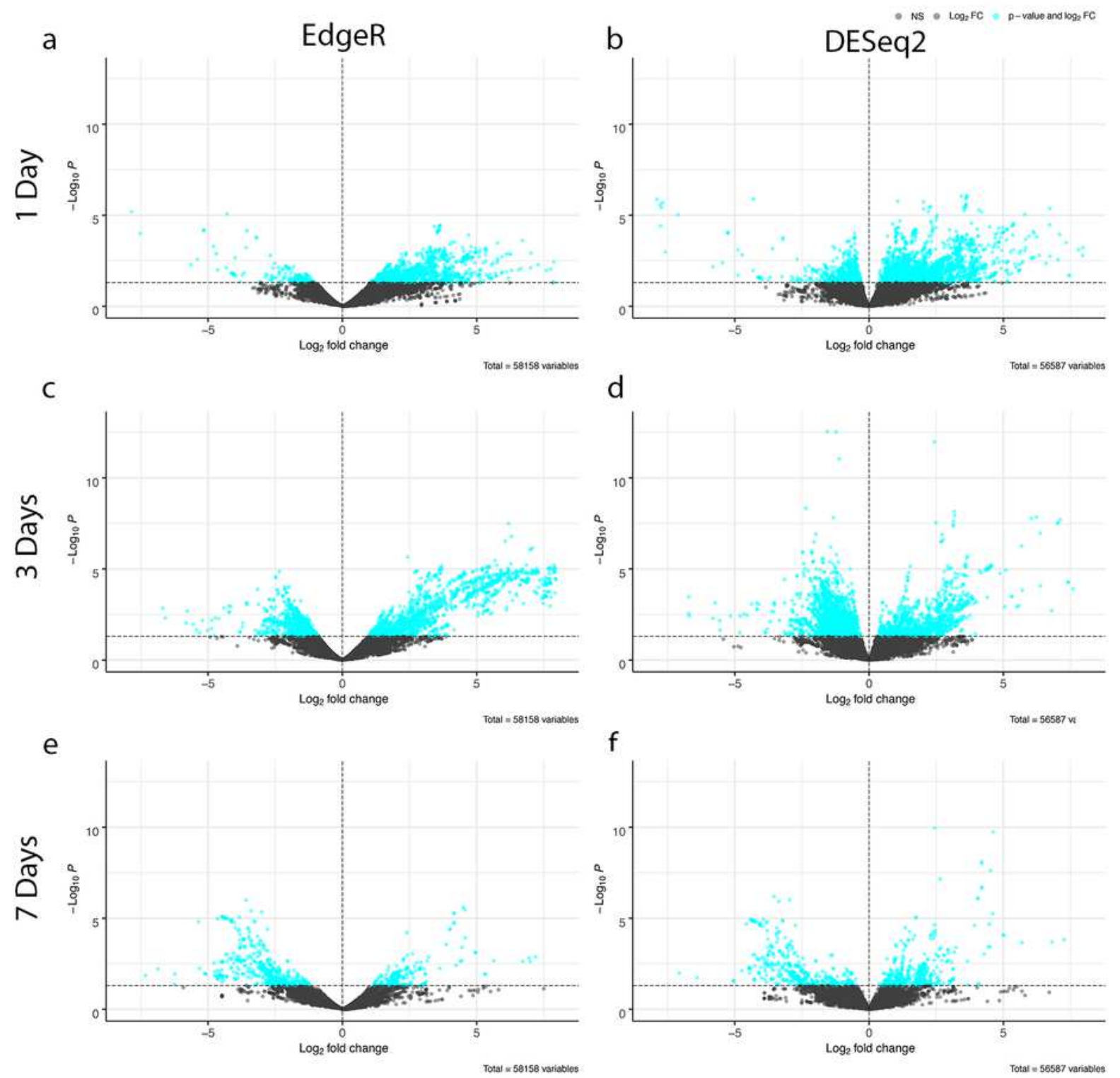

\section{Figure 2}

Volcano plot of differential gene expression in G. bimaculatus prothoracic ganglia at three different time points after deafferentation. The horizontal dotted line marks a p-value of 0.05 , and the vertical dotted line marks no predicted fold change. Each point represents a contig determined to be differentially regulated by EdgeR or DESeq2. Blue points represent the contigs determined to be significantly regulated. 
Upregulated

a

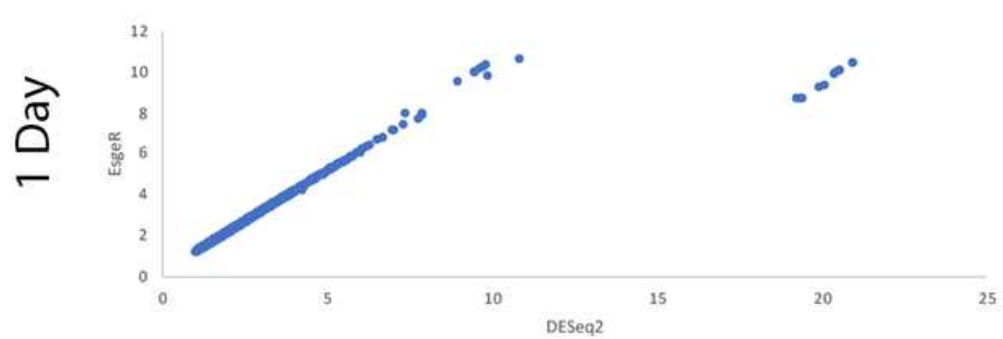

$\mathrm{b}$

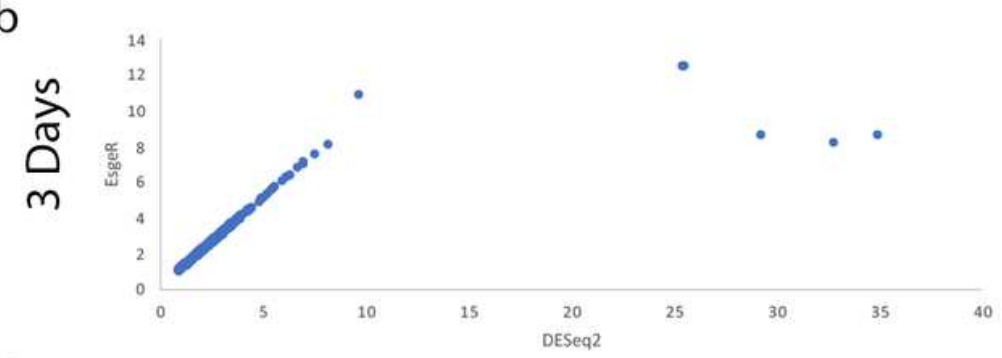

C

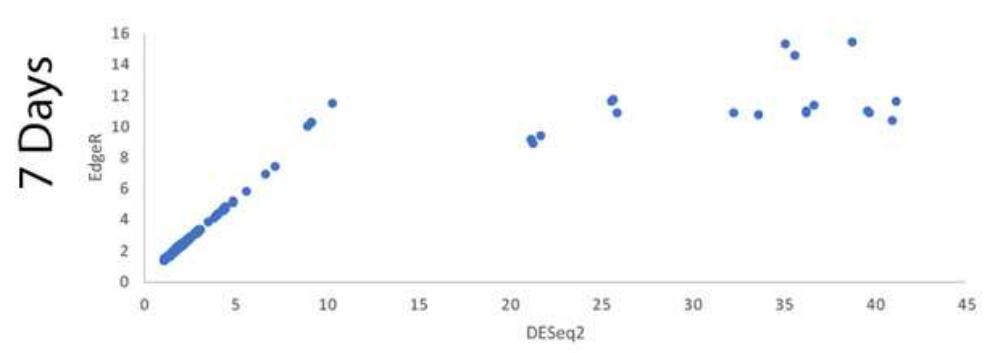

Downregulated

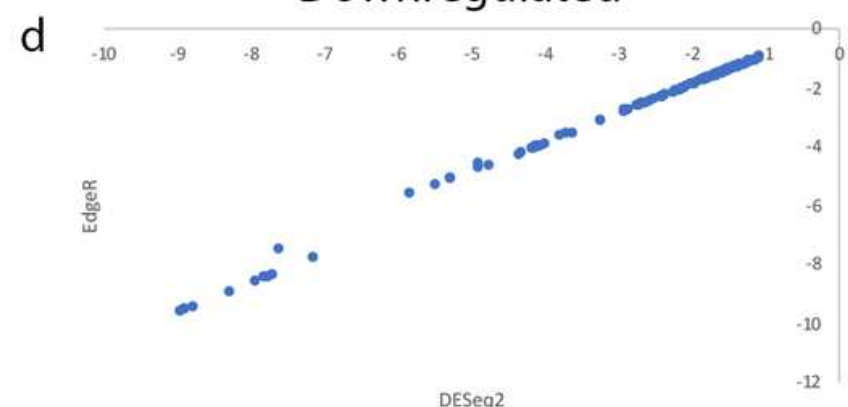

e

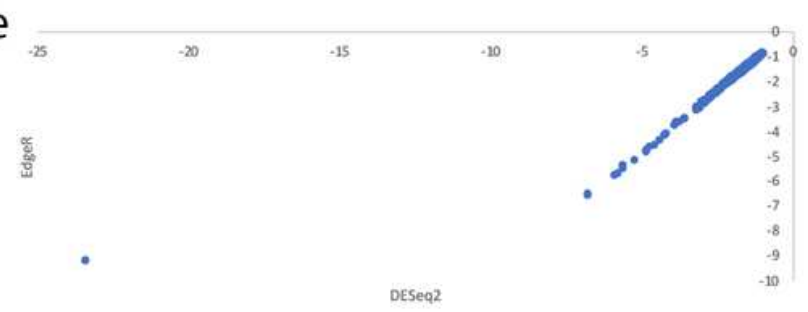

f

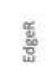

\section{Figure 3}

Correlation of fold-changes predicted by EdgeR and DESeq2 for upregulated transcripts (left) and downregulated transcripts (right) at one, three, and seven days. 

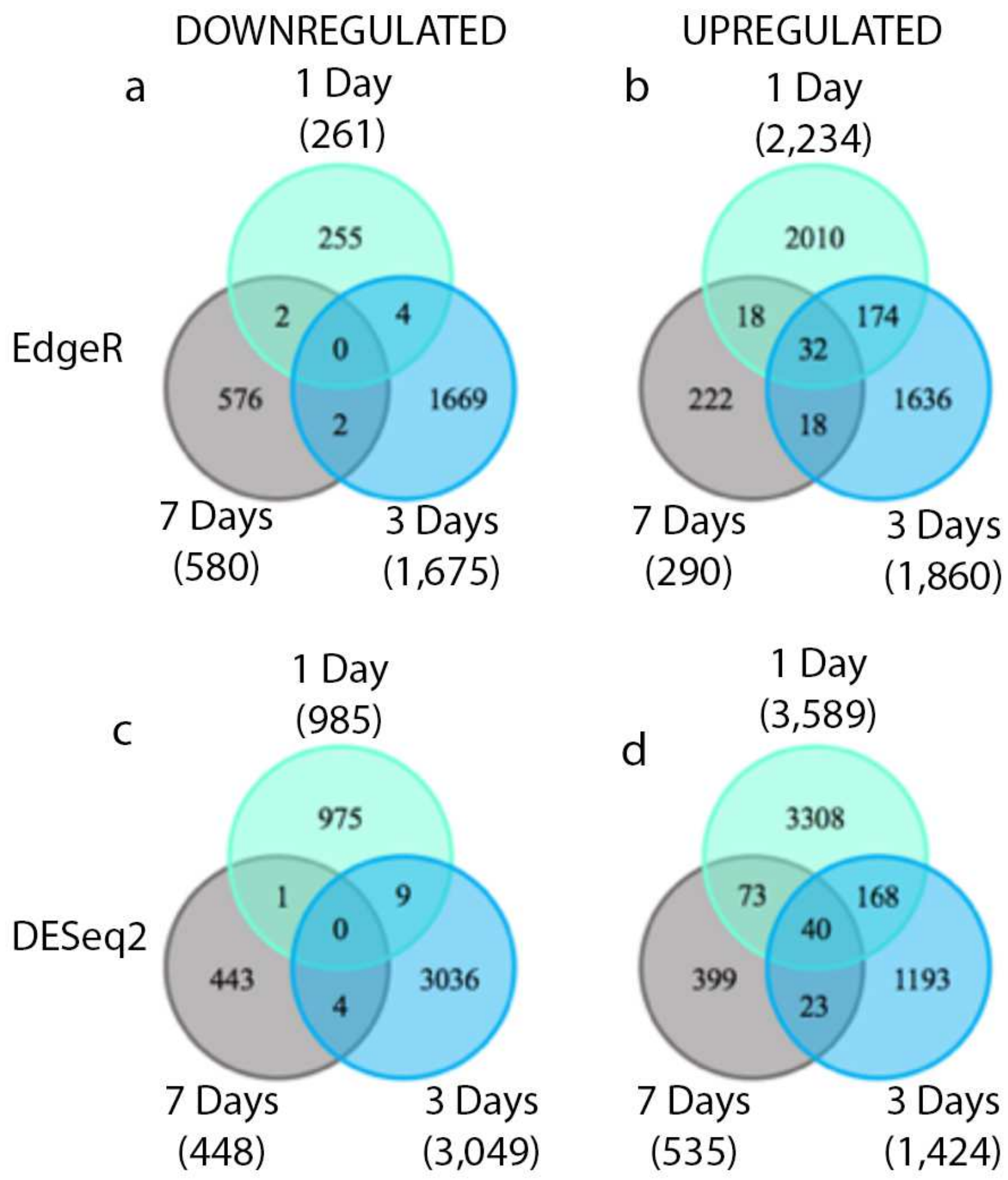

Figure 4

Differentially regulated genes across three time points. Similar patterns in relative numbers of differentially regulated genes are observed between the two programs. a) EdgeR identified downregulated genes b) EdgeR identified upregulated genes c) DESeq2 identified downregulated genes d) DESeq2 identified upregulated genes. 

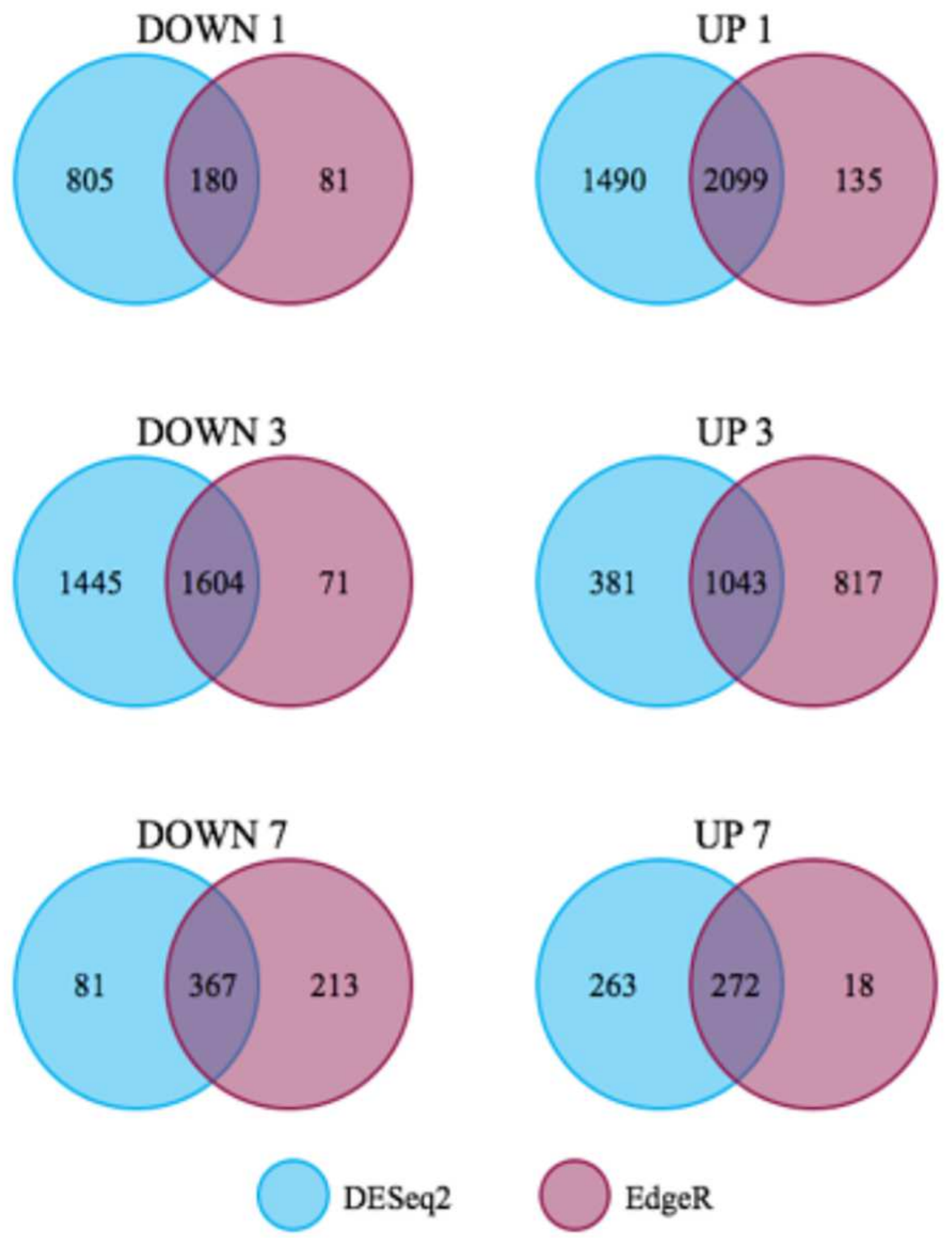

\section{Figure 5}

Differentially regulated genes compared across the two analytical programs, DESeq2 and EdgeR. The number of genes found to be differentially regulated by both programs varies by condition. 


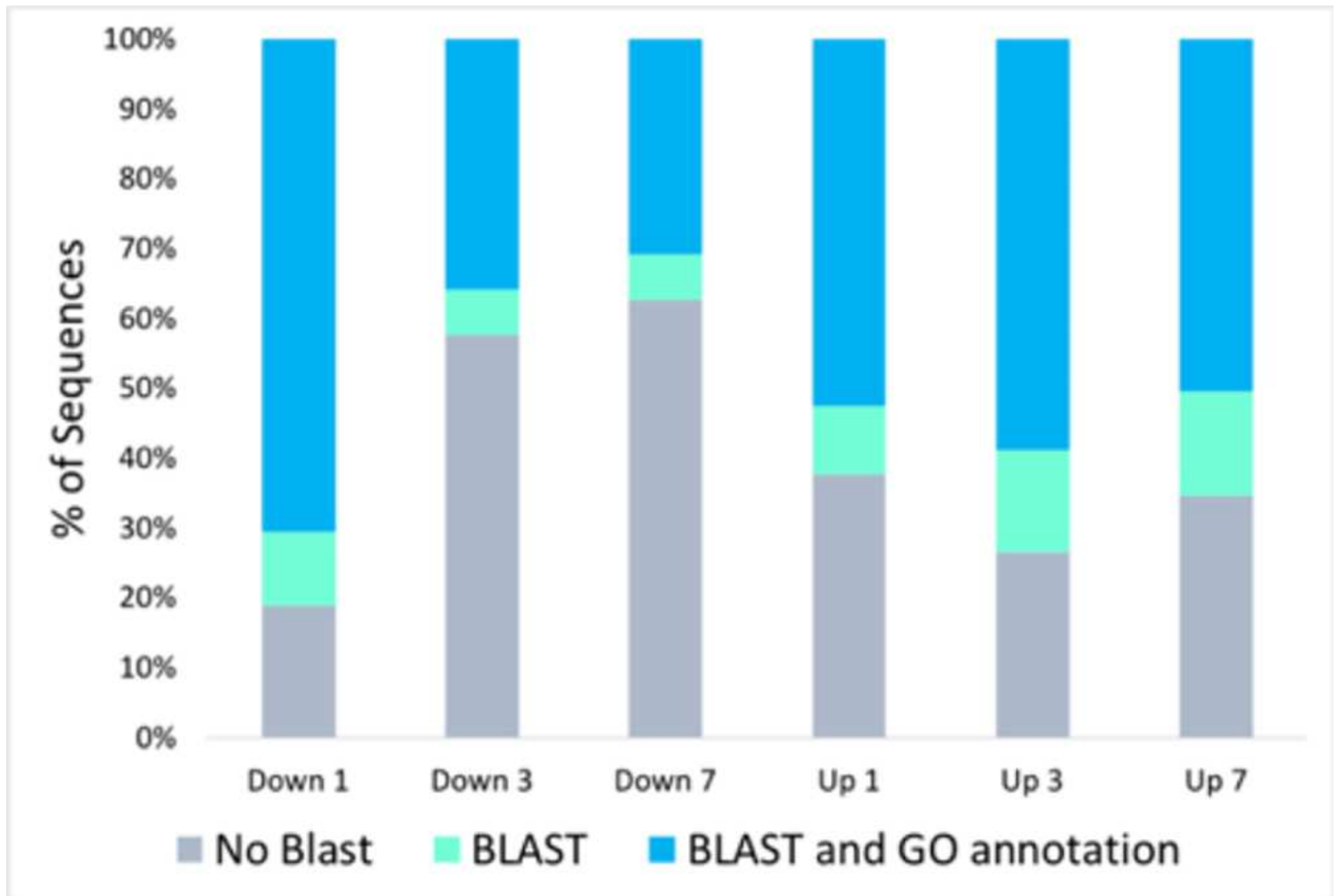

Figure 6

Percentage of sequences with no BLAST hits, BLAST hits, and BLAST hits with additional GO term mapping. Distribution of sequences varies across times points and regulation. 


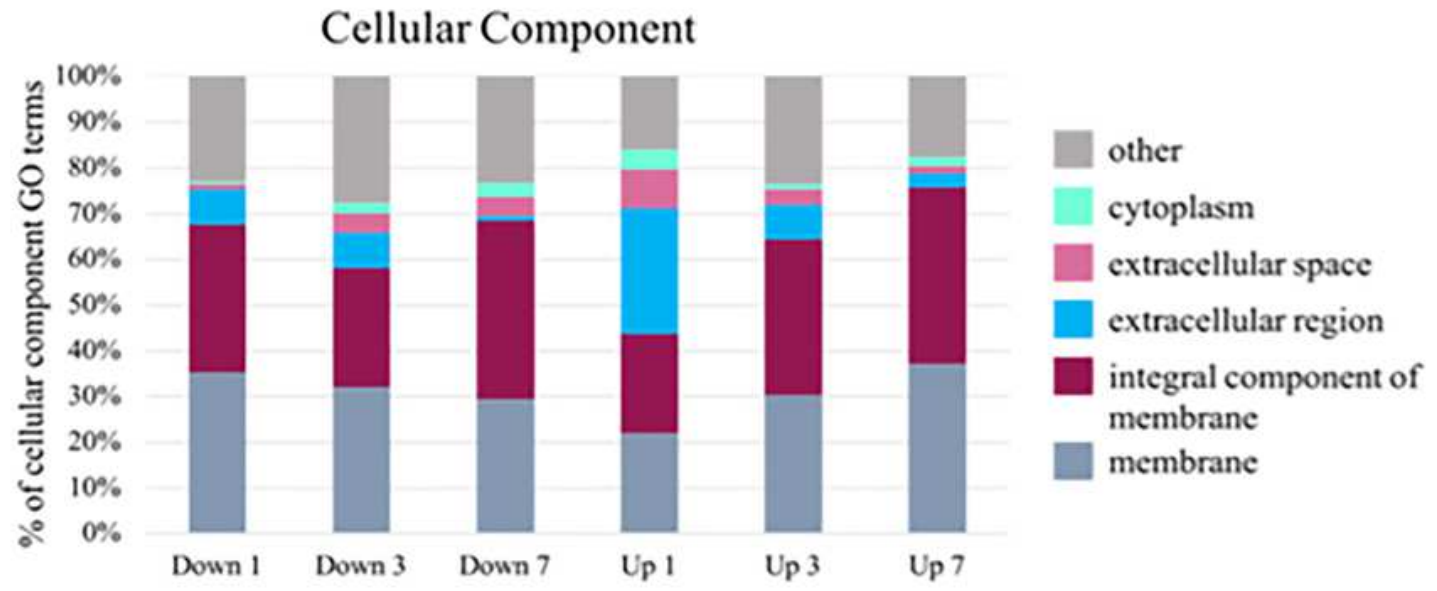

Molecular Function

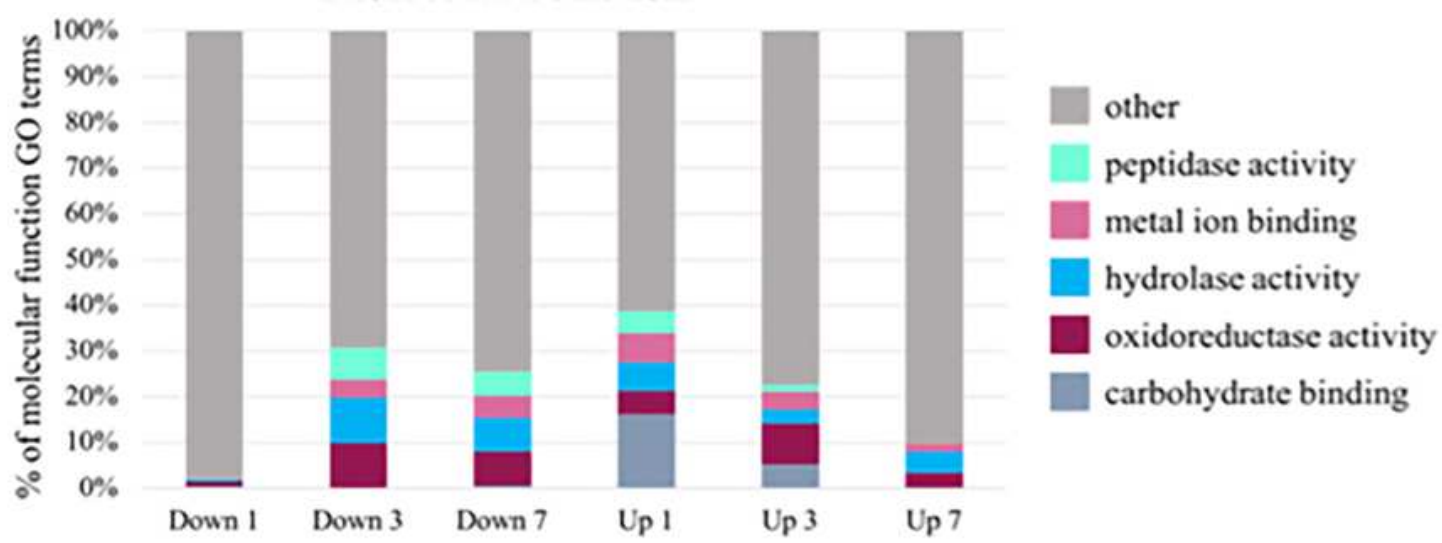

Biological Process

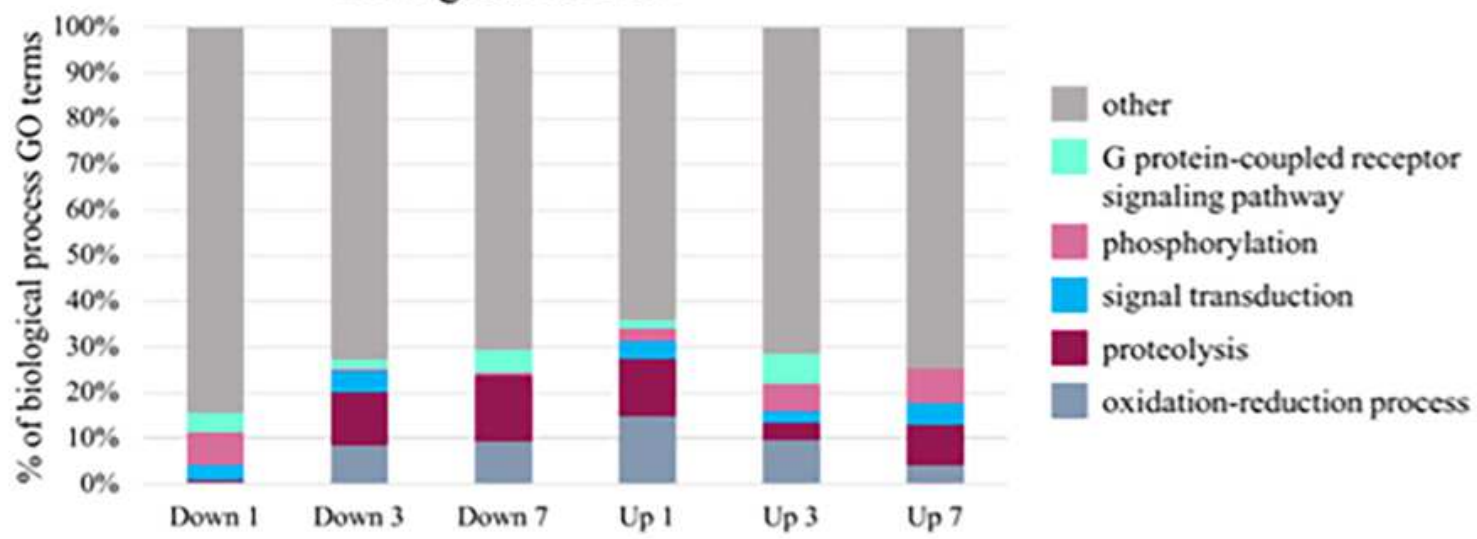

Figure 7

GO term analysis organized into the three root classes: cellular component (CC), molecular function (MF), and biological process (BP). The top 5 represented $\mathrm{GO}$ terms across all time points in each class are represented. Many highly represented GO terms were found in the CC class whereas a broader range of GO terms were found in the MF and BP classes. 

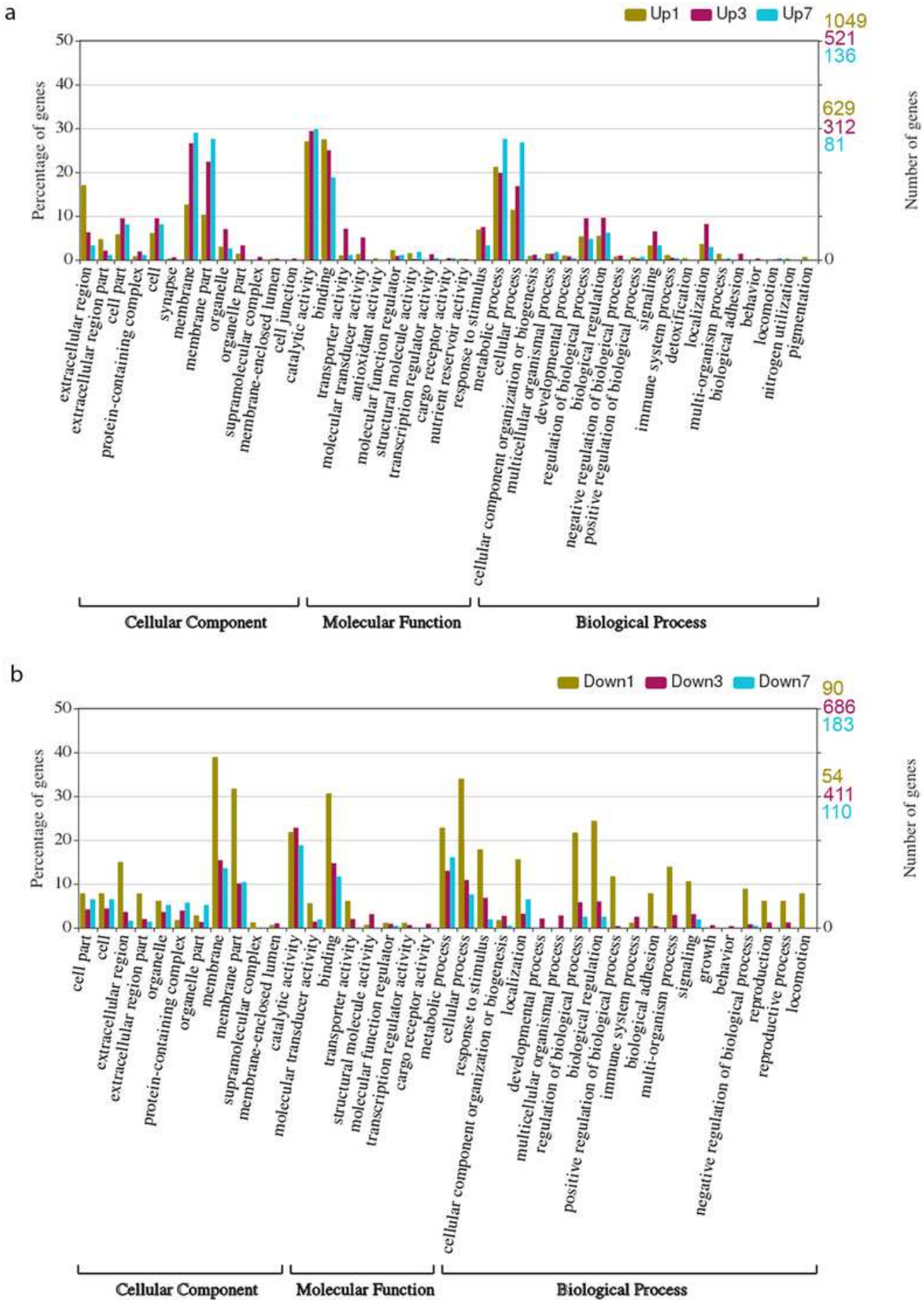

\section{Figure 8}

WEGO histograms with the distribution of Gene Ontology terms grouped by cellular component, molecular function, or biological process. a) GO terms associated with downregulated genes across all time points. b) GO terms associated with upregulated genes across all time points. Percentages are noted on the left and the number of genes within the given list that were annotated with the GO term/child term are noted on the right. On the right axis, the top numbers (olive) corresponds to the one-day data, the 
middle numbers, (magenta) corresponds to the three-day data, and the bottom numbers correspond to the seven-day data (teal).

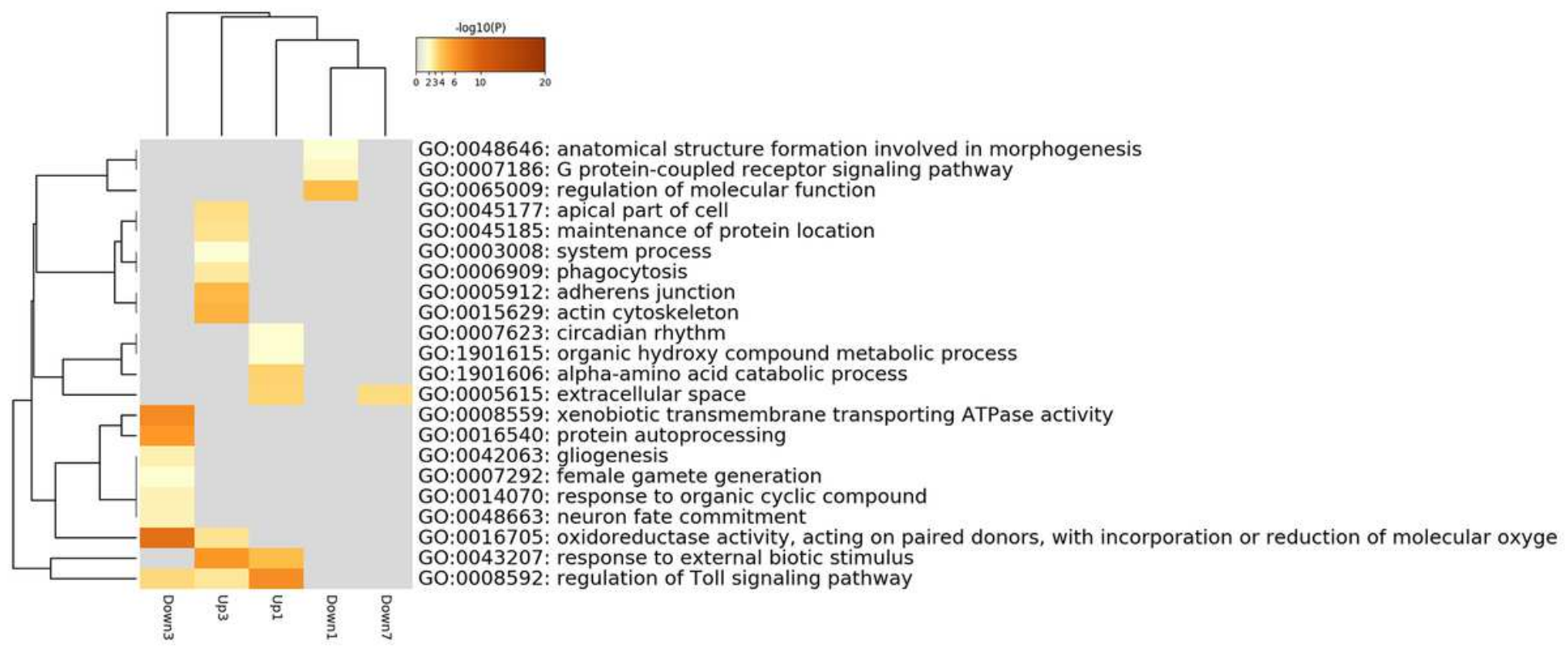

Figure 9

Heatmap of enrichment terms as determined by $M$ etascape. Colored by $p$-value as indicated at the top.

\section{Supplementary Files}

This is a list of supplementary files associated with this preprint. Click to download.

- SupplementalMaterialsTitlePage.pdf 